\title{
A Single-Phase Modelling for the Oxygen Uptake Rate in Excess Post-Exercise Oxygen Consumption
}

\section{Maria Carolina Traina Gama}

Universidade Estadual de Campinas - Unicamp

Claudio Alexandre Gobatto

Universidade Estadual de Campinas - Unicamp

Dimas Roberto Vollet ( $\nabla$ dr.vollet@unesp.br)

Universidade Estadual Paulista - Unesp

\section{Research Article}

Keywords: dVO2/dt, WEP, EPOC, physiological parameter, VO2

Posted Date: February 16th, 2021

DOI: https://doi.org/10.21203/rs.3.rs-220974/v1

License: (c) (i) This work is licensed under a Creative Commons Attribution 4.0 International License.

Read Full License 


\section{A Single-Phase Modelling for the Oxygen Uptake Rate in Excess Post- 2 Exercise Oxygen Consumption}

4 Maria Carolina Traina Gama ${ }^{1}$, Claudio Alexandre Gobatto ${ }^{1}$, Dimas

5 Roberto Vollet ${ }^{2, *}$

6

$7 \quad{ }^{1}$ UNICAMP - Universidade Estadual de Campinas, Faculdade de Ciências Aplicadas,

8 Campus de Limeira, Limeira, SP, Brazil.

$92{ }^{2}$ Unesp - Univ Estadual Paulista, IGCE, Departamento de Física, Campus de Rio

10 Claro, Rio Claro, SP, Brazil.

11 *corresponding author: e-mail: $\underline{\text { dr.vollet @ unesp.br }}$

\section{Abstract}

A two-parameters [a rate constant $k$ and a factor $f(0 \leq f<1)$ ] modelling, describing satisfactorily the post-exercise oxygen uptake rate $\left(\mathrm{V}_{\mathrm{O} 2}\right)$ as a function of the recovery time $(t)$, is presented. $f$ controls the rate equation $\mathrm{d} V_{\mathrm{O} 2} / \mathrm{d} t$, particularly at $t=0$ where $\left(\mathrm{d} V_{\mathrm{O} 2} / \mathrm{d} t\right)_{t=0} \propto-k(1-f)$, a less abrupt decay than $\left(\mathrm{d} V_{\mathrm{O} 2} / \mathrm{d} t\right)_{t=0} \propto-k$ expected from an exponential. Fitting the model to a set of experimental $V_{\mathrm{O} 2}$ vs $t$ data after a $3 \mathrm{MT}$ it was found a set of values with $f$ close to 0 and another with $f>1 / 2$, with a narrow distribution of values for the half-recovery time $\tau_{1 / 2}=(1 / k) \ln [(2-f) /(1-f)]\left(\left\langle\tau_{1 / 2}\right\rangle=0.641 \mathrm{~min}, \sigma=0.062\right.$ min), very similar to that $(T)$ found by fitting a model based on a logit transform $(\langle T\rangle=0.672 \mathrm{~min}, \sigma=0.081 \mathrm{~min})$. The parameter $f$ is a reliable index of the initial acceleration of the oxygen uptake rate recovery (and likely of the heart rate recovery) and, together with the half-recovery time $\tau_{1 / 2}$, may be a useful method in characterizing and monitoring performs and exercise forms, very important in the physiology area. 


\section{Introduction}

Three-minute all-out test (3MT) is a maximal effort evaluation protocol based on the original model of critical power whose metabolic parameters of aerobic (EP) and anaerobic (WEP) capacities are obtained through the mathematic analysis of a subject's output power kinetics. The kinetics of output power of 3MT can determine important parameters for training prescription such as some transition limits when it comes to effort intensity ${ }^{1}$. EP is the threshold that measures the transition from intense to severe exercise and uses mainly oxidative metabolism ${ }^{1,2}$, while WEP uses anaerobic sources and is a finite amount of work carried until it runs out in severe intensity exercises ${ }^{3}$. The post-exercise oxygen consumption (EPOC) after 3MT can provide important information about the maximum effort performed by the individual.

Direct physiological parameters such as blood lactate concentration and oxygen uptake measure the domains of intensity transition during exercise ${ }^{4}$. Researchers have been using such physiological markers, collecting them right after exercise, to determine the contribution of the aerobic and anaerobic energetic metabolisms ${ }^{5}$. EPOC may physiologically represent the reestablishment of blood and muscular $\mathrm{O}_{2}$ to ATP and $\mathrm{PCr}$ resynthesis, as well as removing lactate from high intensity efforts ${ }^{6}$. Blood lactate concentration and EPOC can reliably estimate the anaerobic capacity in $3 \mathrm{MT}^{7}$.

The oxygen uptake rate $\left(V_{\mathrm{O} 2}\right)$ recovery is conventionally assumed to proceed in a biphasic manner, consisting of "fast" and "slow" components. The overall process has been described by two simple exponentials and it has been acknowledged as the standing model $^{8-11}$.

However, it has been $\operatorname{argued}^{12}$ that application of two-exponential model to postexercise oxygen uptake rate and heart rate (HR) might be questionable, since metabolic processes like lactate elimination could follow rather the mass action law, instead an exponential decay. Besides that, the procedure by curve fitting to a first order exponential decay in HR recovery, following max exercise, seem not to confirm that a first order equation is the optimal model to establish the most appropriate exercise protocol $^{13}$. The time decaying obtained by this procedure varied unacceptably with small changes in onset of monitoring and the residuals of the fitted curve were nonrandom ${ }^{13}$.

Therefore, it is opportune to consider alternative models based on the mass action law, for instance, to fit the experimental data. The mass action law requires that 
62 the rate equation for $V_{\mathrm{O} 2}$ decaying takes into account two components: the residual

63 "oxygen debt" $\left(V_{\mathrm{O} 2}-V_{\mathrm{O} 2 \mathrm{rest}}\right)$ and the "oxygen debt" already paid ( $\left.V_{\mathrm{O} 2 \mathrm{peak}}-V_{\mathrm{O} 2}\right)$, in a

64 total net peak post-exercise uptake $\left(V_{\text {O2peak }}-V_{\text {O2rest }}\right)^{12}$. Stupnicki et al ${ }^{12}$, by applying a

$65 \operatorname{logit}$ transform, $\log \left[X_{\mathrm{i}} /\left(X_{\text {peak }}-X_{\mathrm{i}}\right)\right]$, to the recorded variables $X_{\mathrm{i}}$ [post-exercise heart rate

$66(\mathrm{HR})$ and $\left.V_{\mathrm{O} 2}\right]$ and relating them to the decimal logarithm of the recovery time $t$,

67 obtained single-phase course of changes for both variables, rendering a linear

68 relationship, which facilitated various comparisons. This logit-log transformation was

69 also used with success to model horse post-exercise heat rate recovery ${ }^{14}$. However,

70 Stupnicki et al. ${ }^{12}$ did not give an analytical form to fit the data nor provided the rate

71 equation for the $V_{\mathrm{O} 2}$ decaying.

72 In this work, the rate equation for the oxygen uptake rate decaying, $\mathrm{d} V_{\mathrm{O} 2} / \mathrm{d} t$, is

73 considered as being first-order on the fraction $X$ of the residual "oxygen debt" to decay, 74 and first-order on $(1-f X)$, a function of the fraction $(1-X)$ of the "oxygen debt" already

75 paid, where $f(0 \leq f<1)$ is a factor introduced to control the recovery start. The

76 approach provides an analytical form for the oxygen uptake rate as a recovery time

77 function, without loss of generality, since, by setting $f=0$, the model becomes just the

78 standing mono-exponential one.

79 Theoretical aspects

80 The $f$-single-phase modelling

81 The rate equation, $\mathrm{d} V_{\mathrm{O} 2} / \mathrm{d} t$, for the oxygen uptake rate $\left(V_{\mathrm{O} 2}\right)$ at the recovery

82 instant $t$ was assumed as being first-order on the fraction $X$ of the residual "oxygen

83 debt" to decay, $X=\left(V_{\mathrm{O} 2}-V_{\mathrm{O} 2 \text { rest }}\right) /\left(V_{\mathrm{O} 2 \text { peak }}-V_{\mathrm{O} 2 \text { rest }}\right)$, where $V_{\mathrm{O} 2 \text { peak }}$ is oxygen uptake rate

84 at $t=0$ and $V_{\mathrm{O} 2 \mathrm{rest}}$ the oxygen uptake rate at rest $(t \rightarrow \infty)$, and first-order on $(1-f X)$, a

85 function of the fraction $(1-X)$ of the "oxygen debt" already paid, $(1-X)=\left(V_{\text {O2peak }}-\right.$

$\left.86 V_{\mathrm{O} 2}\right) /\left(V_{\mathrm{O} 2 \text { peak }}-V_{\mathrm{O} 2 \mathrm{rest}}\right)$, where $f$, with the constraint $0 \leq f<1$, is a factor introduced to

87 control the recovery start. Since $\mathrm{d} V_{\mathrm{O} 2} / \mathrm{d} t \propto \mathrm{d} X / \mathrm{d} t$, it follows

88

$$
\mathrm{d} X / \mathrm{d} t=-k X(1-f X)
$$

where $k\left(\mathrm{~min}^{-1}\right)$ is a rate constant. The parameter $f$ controls the rate equation (equation

90 (1)), particularly at $t=0$ when $X=1$, so

$$
(\mathrm{d} X / \mathrm{d} t)_{t=0}=-k(1-f)
$$


92

93

94

95

96

97

98

99

100

101

102

103

104

105

106

107

108

109

110

111

112

114

115

The solution of equation (1), under the conditions $X=1$ at $t=0$ and $X=0$ at $t \rightarrow$ $\infty$, is

$$
X=\exp (-k t) /\{1-f[1-\exp (-k t)]\}
$$

If $f=0$, the decaying process is a simple exponential $X=\exp (-k t)$ and $(\mathrm{d} X / \mathrm{d} t)_{t=0}=-k$, a more abrupt decaying than $(\mathrm{d} X / \mathrm{d} t)_{t=0}=-k(1-f)$ when $f>0$.

The half-recovery time $\left(\tau_{1 / 2}\right)$, the time for $X$ to decay to half of its initial value, can be evaluated from equation (3) as

$$
\tau_{1 / 2}=(1 / k) \ln [(2-f) /(1-f)]
$$

Restoring the definition $X=\left(V_{\mathrm{O} 2}-V_{\mathrm{O} 2 \text { rest }}\right) /\left(V_{\text {O2peak }}-V_{\mathrm{O} 2 \text { rest }}\right)$ in equation (3) yields

$V_{\mathrm{O} 2}=V_{\mathrm{O} 2 \text { rest }}+\left(V_{\mathrm{O} 2 \text { peak }}-V_{\mathrm{O} 2 \text { rest }}\right) \exp (-k t) /\{1-f[1-\exp (-k t)]\}$.

Equation (5), named here $f$-single-phase ( $f 1 \mathrm{p})$ model, can be fitted to the experimental $V_{\mathrm{O} 2}$ vs $t$ data to obtain the parameters $k, f, V_{\mathrm{O} 2 \text { peak }}$ and $V_{\mathrm{O} 2 \text { rest. Equivalently, equation (5) }}$ could be expressed in terms of the parameters $k$ and $\tau_{1 / 2}$, since $f$ can be solved from equation (4) as $\left.f=\left[\exp \left(k \tau_{1 / 2}\right)-2\right] /\left[\exp \left(k \tau_{1 / 2}\right)-1\right)\right]$, with the constraint $k \tau_{1 / 2} \geq \ln 2$.

Therefore, among the parameters $k, f$ and $\tau_{1 / 2}$, only two of them are independent in the flp model. by the quantity ( $\left.V_{\text {O2peak }}-V_{\text {O2rest }}\right)$, represents the total net volume of oxygen uptake up to the instant $t, V T_{\mathrm{O} 2 \mathrm{net}}(t)$, excluded the contribution of the rest component. $X_{\text {integ }}(t)$ can be evaluated by

$$
X_{\text {integ }}(t)=-(1 / k)(1 / f) \ln \{1-f[1-\exp (-k t)]\},
$$

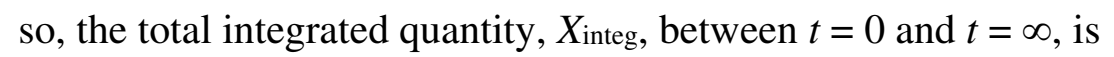

$$
X_{\text {integ }}=-(1 / k)(1 / f) \ln (1-f)=-\tau_{1 / 2}(1 / f) \ln (1-f) / \ln [(2-f) /(1-f)] .
$$


116 The constraint equation (4) has been used to write $X_{\text {integ }}$ indistinctly in terms of $k$ and $f$

117 or $\tau_{1 / 2}$ and $f$ in equation (7). If $f=0$, equation (7) becomes $X_{\text {integ }}=1 / k=\tau_{1 / 2} / \ln 2$ just as in

118 case of an exponential decay, since the limit of $(1 / f) \ln [(1-f)]$ is -1 when $f \rightarrow 0$.

119 The total net volume of oxygen uptake up to complete recovery $(t=\infty), V T_{\text {o2net, }}$

120 the so-called EPOC, is obtained by multiplying equation (7) by the quantity ( $V_{\text {ozpeak }-}$

121 Vo2rest), or

$$
V T_{\mathrm{O} 2 \text { net }}=\left(V_{\mathrm{O} 2 \text { peak }}-V_{\mathrm{O} 2 \mathrm{rest}}\right) X_{\text {integ }} \text {. }
$$

To better illustrate the effect of the parameter $f$ on the experimental $V_{\mathrm{O} 2}$ vs $t$

curve, Figure 1 shows plots of equation (3) for several values of $k$ (or $f$ ) for a fixed value of $\tau_{1 / 2}=\ln 2$. The inclination $(\mathrm{d} X / \mathrm{d} t)_{t=0}=-k(1-f)$ at $t=0$ is effectively less abrupt with increasing $f$ (insert in Figure 1), and particularly less abrupt than an exponential decay where $(\mathrm{d} X / \mathrm{d} t)_{t=0}=-k$. The increase of the parameter $f$ retards effectively the decaying of $V_{\mathrm{O} 2}$ at the beginning of the recovery, however, the rate of decaying at $t=\tau_{1 / 2},(\mathrm{~d} X / \mathrm{d} t)_{\tau / 12}$ $=-(k / 2)[1-f / 2]$, becomes more abrupt with increasing $f$, as shown by the tangent dash

130 lines plotted with slopes $(\mathrm{d} X / \mathrm{d} t)_{\tau_{1 / 2}}$ at $t=\tau_{1 / 2}$ in Figure 1 for several values of $k$ (or $f$, since $\tau_{1 / 2}=$ cte $)$.

\section{About the logit transformation}

It is opportune to compare the present $f 1 \mathrm{p}$ model with that could be inferred

136 from a graphical procedure proposed by Stupnicki et al. ${ }^{12}$. They applied a logit

137 transformation, $\log \left[X_{\mathrm{i}} /\left(X_{\text {peak }}-X_{\mathrm{i}}\right)\right]$, to the net recorded variables $X_{\mathrm{i}}\left(X_{\mathrm{i}}=V_{\mathrm{O} 2}-V_{\mathrm{O} 2 \text { rest }}\right.$ and

$\left.138 X_{\text {peak }}=V_{\text {O2peak }}-V_{\text {O2rest }}\right)$, and observed an unique regime of linear plot, with a negative

139 slope $(-\alpha)$, in the whole domain of the logarithm of the recovery time $t$. Then, the

140 relation $\log \left[X_{\mathrm{i}} /\left(X_{\text {peak }}-X_{\mathrm{i}}\right)\right]=-\alpha \log t+B$, where $B$ is a constant, should be kept. By

141 writing $B=\log T^{\alpha}$, where $T$ is a characteristic time, it is concluded that $X_{\mathrm{i}}=$

$142 X_{\text {peak }}\left\{1 /\left[(t / T)^{\alpha}+1\right]\right\}$, or 
144 to keep the same notation for the fraction $X=X_{\mathrm{i}} / X_{\text {peak }}=\left(V_{\mathrm{O} 2}-V_{\mathrm{O} 2 \text { rest }}\right) /\left(V_{\mathrm{O} 2 \text { peak }}-V_{\mathrm{O} 2 \text { rest }}\right)$

145 of the present work. So equation (9) could be written as

$$
V_{\mathrm{O} 2}=V_{\mathrm{O} 2 \text { rest }}+\left(V_{\mathrm{O} 2 \text { peak }}-V_{\mathrm{O} 2 \mathrm{rest}}\right)\left\{1 /\left[(t / T)^{\alpha}+1\right]\right\} .
$$

147 Equation (10), named here Stu model, could be fitted to the experimental Vo2 vs $t$ data

148 and the parameters $\alpha, T, V_{\mathrm{O} 2 \text { peak }}$ and $V_{\mathrm{O} 2 \text { rest }}$ determined. $T$ represents the half-recovery

149 time, the time for $X$ in equation (9) to decay to $1 / 2$, the same meaning of the parameter $150 \tau_{1 / 2}$ of the $f 1 \mathrm{p}$ model.

151 The rate equation that governs the decaying law of equation (9) is given by

$$
\mathrm{d} X / \mathrm{d} t=-(\alpha / T) X^{(1+1 / \alpha)}(1-X)^{(1-1 / \alpha)}
$$

being, therefore, of $(1+1 / \alpha)$-order on $X$ and $(1-1 / \alpha)$-order on $(1-X)$. The overall order with respect to both, $X$ and $(1-X)$, remains constant and equal to $(1-1 / \alpha)+(1+1 / \alpha)=2$, independent on $\alpha$. The parameter $\alpha$ should be $\geq 1$ to avoid negative-order on $(1-X)$. With $\alpha>1$, equation (11) gives always $(\mathrm{d} X / \mathrm{d} t)_{t=0}=0$ at $t=0$, which is different from $(\mathrm{d} X / \mathrm{d} t)_{t=0}=-k(1-f)$ of the $f 1 \mathrm{p}$ model. If $\alpha=1$, the rate equation (11) would be a secondorder on $X$ and zero-order on $(1-X)$, and $(\mathrm{d} X / \mathrm{d} t)_{t=0}=-(1 / T)$ at $t=0$, as the unique exception.

\section{Material and methods}

Ten recreationally-trained male runners $(24 \pm 4$ years; $80.3 \pm 8.7 \mathrm{~kg} ; 179 \pm 5$ $\mathrm{cm} ; 9.0 \pm 2.5 \%$ body fat) participated in this study performing $3 \mathrm{MT}$, after signing an informed consent form approved by the Human Research Ethics Committee of the University of Campinas (protocol CAAE: 61934516.5.0000.5404), in accordance with the Declaration of Helsinki.

3MT was carried out on a non-motorized treadmill (NMT) after a five-minute warm-up consisting in walking on a motorized treadmill (MT) at $6 \mathrm{~km} / \mathrm{h}$. Each participant ran on a NMT tethered to an adjustable-height pole by a steel cable attached to a load cell (CSL/ZL-250, MK Control and Instrumentation) ${ }^{15}$. Despite participants were constantly encouraged during the test, no information in regards of time was given. The test only was considered successful after 3 minutes of non-stoppable

172 running. The mechanical output power generated by each subject during the $3 \mathrm{MT}$ test 
173 was recorded and analyzed against time to find the aerobic capacity (EP) and anaerobic work capacity (WEP) values of the power output graph ${ }^{2}$.

The oxygen uptake and carbon dioxide production were measured by using a gas analyzer (Cosmed Italy $\mathrm{K} 4 \mathrm{~b}^{2}$ ) that was calibrated after each session. The gas analyzer was integrated to an online system of breath-by-breath data caption. The oxygen uptake rate $\left(V_{\mathrm{O} 2}\right)$ was measured as a function of the recovery time $(t)$ up to about 10 min after the start of the $3 \mathrm{MT}$ recovery. The models were fitted to the experimental $V_{\mathrm{O} 2}$ vs $t$ data.

The fitting processes were carried out using a routine of least-squares method based on the Levenberg-Marquardt algorithm. When was the case, the similarity between the parameters given by different models was statistically tested for equal means using One-Way ANOVA.

\section{Results}

Figure 2 shows the $f 1 \mathrm{p}$ model fitting well the experimental $V_{\mathrm{O} 2} \mathrm{vs} t$. The fitted parameters $V_{\text {O2peak, }} V_{\text {O2rest }}, k$, and $f$ (or $\tau_{1 / 2}$ ) are shown in Table 1 ( $f 1 \mathrm{p}$ model) with the respective standard errors. The data were organized in order of increasing $f$. When the fitting process converged rapidly to the lower constraint value $f=0$ (subjects 1 and 2 in Table 1), $f$ was set to 0 and $k$ obtained by fitting, and $\tau_{1 / 2}$ evaluated through $\tau_{1 / 2}=$ $(1 / k) \ln 2$. The mean value of $\tau_{1 / 2}$ was $\left\langle\tau_{1 / 2}\right\rangle=0.641$ min with standard deviation $\sigma=$ $0.062 \mathrm{~min}$.

(insert Figure 2) (insert Table 1)

Figure 3(a) shows the fitting of the Stu model to the experimental data of the subject number 8 , as an instance, in comparison with that of the $f 1 \mathrm{p}$ model. The Stu model fitted well all the present set of experimental data. Table 1 (Stu model) shows the fitted parameters $V_{\mathrm{O} 2 \text { peak, }} V_{\mathrm{O} 2 \mathrm{rest}}, \alpha$, and $T$ with the standard errors. Figure 3(b) shows the adjusted $R$-square and the reduced sum of squares $\chi^{2}$ obtained by fitting both the models (Stu and $f 1 \mathrm{p}$ ) to the experimental data. The mean value of the half-recovery time $T$ was $\langle T\rangle=0.672 \mathrm{~min}$ with standard deviation $\sigma=0.081 \mathrm{~min}$. Figure 3(c) shows the distribution of $T$ and $\tau_{1 / 2}$ plotted as normal Gaussian distributions $F(\tau, \sigma)=$ $\left[1 /\left(2 \pi \sigma^{2}\right)^{1 / 2}\right] \exp \left[-((t-\tau) / \sigma)^{2} / 2\right]$, using the mean values $\left\langle\tau_{1 / 2}\right\rangle$ and $\langle T\rangle$ with the respective standard deviations $(\sigma)$. 
The standard biphasic two-exponential model, which can be cast as $V_{\mathrm{O} 2}=V_{\mathrm{O} 2 \mathrm{rest}}$

$206+A_{1} \exp \left(-t / \tau_{1}\right)+A_{2} \exp \left(-t / \tau_{2}\right)$, where $A_{1}$ and $A_{2}$ are constants and $\tau_{1}$ and $\tau_{2}$ are the "fast"

207 and the "slow" decaying constant times, respectively, was also fitted to the present

208 experimental data. In order to minimize the parameters to be fitted, Vo2rest was fixed as

209 the mean value of $V_{02}$ in the last minute of recovery and the parameters $A_{1}, \tau_{1}, A_{2}$, and

$210 \tau_{2}$ were obtained by fitting. Table 1 (two-exponential model) shows the fitted

211 parameters with the standard errors.

212 The constant times $\tau_{1}$ and $\tau_{2}$ were yet converted (in order to compare them with

$213 \tau_{1 / 2}$ ) to the half-recovery times $\tau_{1 / 2}{ }^{(1)}$ and $\tau_{1 / 2}{ }^{(2)}$ by using $\tau_{1 / 2}{ }^{(1)}=\tau_{1} \ln 2$ and $\tau_{1 / 2}{ }^{(2)}=\tau_{2} \ln 2$.

214 The mean values for $\tau_{1 / 2}{ }^{(1)}$ and $\tau_{1 / 2}{ }^{(2)}$ were $\left\langle\tau_{1 / 2}{ }^{(1)}\right\rangle=0.476$ min, with standard deviation

$215 \sigma=0.082 \mathrm{~min}$, and $\left\langle\tau_{1 / 2}{ }^{(2)}\right\rangle=3.94 \mathrm{~min}$, with standard deviation $\sigma=1.91 \mathrm{~min}$.

216 Figure 4(a) shows the reduced residual sum of square $\left(\chi^{2}\right)$ and the adjusted $R$ -

217 square values obtained by fitting the standard biphasic two-exponential model and the

$218 f 1 \mathrm{p}$ model. Figure 4(b) shows normal Gaussian distributions $F(\tau, \sigma)=$

$219\left[1 /\left(2 \pi \sigma^{2}\right)^{1 / 2}\right] \exp \left[-((t-\tau) / \sigma)^{2} / 2\right]$ plotted by using the mean value $\left\langle\tau_{1 / 2}\right\rangle(f 1 \mathrm{p}$ model $)$ and

220 the mean values $\left\langle\tau_{1 / 2}{ }^{(1)}\right\rangle$ and $\left\langle\tau_{1 / 2}{ }^{(2)}\right\rangle$ (standard biphasic model), with the respective

221 standard deviations $(\sigma)$.

$222 \quad$ (insert Figure 4)

\section{Discussion}

The values of $f$ obtained by fitting the $f 1 \mathrm{p}$ model define a group of individuals for which $f$ was zero or lightly greater than zero [subjects 1 to 5 , Table 1 ( $f 1 \mathrm{p}$ model)] and another for which $f>1 / 2$ [subjects 6 to 10 , Table 1 ( $f 1 \mathrm{p}$ model)]. $f>0$ means that the rate equation at $t=0\left[(\mathrm{~d} X / \mathrm{d} t)_{t=0}=-k(1-f)\right]$ is not so abrupt as in case of an exponential. This apparent bimodal distribution of $f$ in this set of recreationally trained subjects may be characteristic of the individuals. Though this hypothesis could not be conclude by analyzing only this set of experimental data, it is clear that the present method opens large possibilities of investigation about exercises and individual performs through the parameter $f$.

Figure 5(a) shows that the values of $\tau_{1 / 2}$ obtained by the $f 1 \mathrm{p}$ model, when analyzed as function of the parameter $f$, are randomly distributed along the horizontal line described by $\tau_{1 / 2}=\left\langle\tau_{1 / 2}\right\rangle=0.641 \mathrm{~min}$ (within the standard error $\sigma=0.062 \mathrm{~min}$ ). 
236 Indeed, the linear fitting of $\tau_{1 / 2}$ vs $f$ in Figure 5(a) yields the fairly horizontal line given

237 by $\tau_{1 / 2}(\min )=0.644-0.0066 \times f$. The constancy of $\tau_{1 / 2}$ may mean a characteristic of the 238 exercise, opening other possibilities for further investigations. Under constant $\tau_{1 / 2}, k=$ $239\left(1 / \tau_{1 / 2}\right) \ln [(2-f) /(1-f)]$, which follows from equation (4), also fitted well to the 240 experimental $k$ vs $f$ data [Figure 5(b)], yielding $\tau_{1 / 2}=0.636$ min with standard error $\sigma=$ $2410.022 \mathrm{~min}$, in good agreement with $\left\langle\tau_{1 / 2}\right\rangle=0.641 \mathrm{~min}$. In addition, $X_{\text {integ }}$ (the second part 242 of equation (7)) also fitted reasonably the experimental $X_{\text {integ }} \operatorname{vs} f$ data with constant $\tau_{1 / 2}$ 243 [Figure 5(c)], yielding $\tau_{1 / 2}=0.646 \mathrm{~min}$ with standard error $\sigma=0.122 \mathrm{~min}$, also in good agreement with $\left\langle\tau_{1 / 2}\right\rangle$.

Figure 5(c) means that $X_{\text {integ }}$ diminishes effectively with the increase of $f$, and so $V T_{\mathrm{O} 2 \mathrm{net}}$, except by the amplitude factor $\left(V_{\mathrm{O} 2 \text { peak }}-V_{\mathrm{O} 2 \mathrm{rest}}\right)$ in equation $(8)$. By keeping higher the rate $V_{\mathrm{O} 2}$ at the beginning of the recovery, which means higher $f$, the total integrated oxygen uptake is paid faster at the beginning of the process. This statement could be supported by computing the time $\tau_{\mathrm{Q}}$ necessary for $X_{\mathrm{integ}}(t)$ (equation (6)) to reach the fraction $[1-\exp (-1)](\sim 63 \%)$ of the total $X_{\text {integ }}$ (equation (7)). Evaluating equation (6) for $t=\tau_{\mathrm{Q}}$ and rending it to [1-exp(-1)] $X_{\text {integ }}$ yields

$$
\tau_{\mathrm{Q}}=-t_{1 / 2} \ln \{[(1-f)[1-\exp (-1)]-(1-f)] / f\} / \ln [(2-f) /(1-f)]
$$

253 Equation (12) fitted reasonably well the experimental $\tau_{\mathrm{Q}}$ vs $f$ data with constant $t_{1 / 2}$

254 [Figure 5(d)], yielding $\tau_{1 / 2}=0.648 \mathrm{~min}$ and standard error $\sigma=0.135 \mathrm{~min}$, also in good agreement with $\left\langle\tau_{1 / 2}\right\rangle$.

The narrow distribution of $t_{1 / 2}$ in the $f 1 \mathrm{p}$ model $\left(\left\langle\tau_{1 / 2}\right\rangle=0.641 \mathrm{~min}, \sigma=0.062\right.$ $\min )$ is in agreement with that of $T$ in the Stu model $(\langle T\rangle=0.672 \mathrm{~min}, \sigma=0.081 \mathrm{~min})$ [Figure 3(c)]. The distributions for $t_{1 / 2}$ and $T$ were statistically tested for equal means using One-Way ANOVA yielding $F=0.9223$ and $p=0.3496$, which means that, at the 0.05 level, the population means are not significantly different. However, the Stu model

261 fitted a bit better the experimental $V_{\mathrm{O} 2}$ vs $t$ data [minor $\chi^{2}$ and better $R$ values in Figure 3(b)]. Nevertheless, the integration of equation (9) from $t=0$ up to $t=T_{\mathrm{f}}$ (the experimental final time of measurement) to rend $X_{\text {integ }}$ by the Stu model (in analogy to equation (7) for the f1p model) gives a complex result: $T_{\mathrm{f} H y p e r g e o m e t r i c} \mathrm{~F} 1[1,1 / \alpha$, $\left.1+1 / \alpha,-\left(T_{\mathrm{f}} / T\right)^{\alpha}\right]$, where Hypergeometric $2 \mathrm{~F} 1\left[1,1 / \alpha, 1+1 / \alpha,-\left(T_{\mathrm{f}} / T\right)^{\alpha}\right]$ is a complex function evaluable numerically only in advanced routines. 
Figure 4(a) shows that $\chi^{2}$ of the $f 1 \mathrm{p}$ model were larger than those of the standard two-exponential model for individuals of the group with $f=0$ or lightly greater than zero (subjects 1 to 5), while $\chi^{2}$ were found minor than those of the standard model for the group of individuals with $f>1 / 2$ (subjects 6 to 10). The same general aspects of quality apply to the $R$-square values from fitting both models (Figure 4(a)). This suggests that the standard two-exponential model could be not quite appropriated to fit experimental data that exhibit a not so high decaying of the rate $V_{\mathrm{O} 2}$ at the beginning of recovery, where the $f 1 \mathrm{p}$ model previews a less abrupt decaying $\left[(\mathrm{d} X / \mathrm{d} t)_{t=0}=-k(1-f)\right]$ when $f>0$. This seems to be the case for the recovery following peak exercise, for which a first order equation is far to confirm to be the optimal model to establish the most appropriate exercise protocol ${ }^{13}$.

Apart from the fact that the standard two-exponential model to have more parameters to be fitted with respect to the single-phase models ( $11 \mathrm{p}$ or Stu), Figure 5(b) shows that the distribution of the "fast" half-recovery time $\tau_{1 / 2}{ }^{(1)}$ was even larger $\left(\left\langle\tau_{1 / 2}^{(1)}\right\rangle=0.476 \mathrm{~min}, \sigma=0.082 \mathrm{~min}\right)$ than $\tau_{1 / 2}$ of the $f 1 \mathrm{p}$ model, and the distribution of the "slow" half-recovery time $\tau_{1 / 2}{ }^{(2)}$ was too large $\left(\left\langle\tau_{1 / 2}{ }^{(2)}\right\rangle=3.94 \mathrm{~min}, \sigma=1.91 \mathrm{~min}\right)$, likely meaningless, even by fixing the rest parameter $V_{\mathrm{O} 2 \mathrm{rest}}$ in the fitting process.

\section{Conclusions}

The present $f$-single-phase modelling is a simple and powerful method able to fit satisfactorily with only two parameters, a rate constant $(k)$ and a factor $f(0 \leq f<1)$, experimental data of oxygen uptake rate in recovery process. The parameter $f$ controls the rate equation $\mathrm{d} V_{\mathrm{O} 2} / \mathrm{d} t$, particularly at $t=0$ where $\left(\mathrm{d} V_{\mathrm{O} 2} / \mathrm{d} t\right)_{t=0} \propto-k(1-f)$, a decay effectively less abrupt than $\left(\mathrm{d} V_{\mathrm{O} 2} / \mathrm{d} t\right)_{t=0} \propto-k$, expected from an exponential. Fitting the modelling to a set of experimental $V_{\mathrm{O} 2} \mathrm{vs} t$ data after a $3 \mathrm{MT}$ yielded a bimodal set of values for $f$ (a set with $f=0$ or close to zero and a set with $f>1 / 2$ ) and a narrow distribution of values for the half-recovery time $\tau_{1 / 2}=(1 / k) \ln [(2-f) /(1-f)]$, with a mean value $\left\langle\tau_{1 / 2}\right\rangle=0.641 \mathrm{~min}$ and standard deviation $\sigma=0.062 \mathrm{~min}$. The distribution of $\tau_{1 / 2}$ was very similar to that found for the half-recovery time $T(\langle T\rangle=0.672 \mathrm{~min}, \sigma=0.081 \mathrm{~min})$ obtained by fitting a single-phase model, inferred from a graphical procedure based on a $\operatorname{logit}-\log$ transformation. The parameter $f$ is a reliable index of the initial acceleration of the oxygen uptake rate recovery (and likely of the heart rate recovery) and, together with the half-recovery time $\tau_{1 / 2}$, may be a useful method in characterizing and 
299 monitoring performs and exercise forms.

\section{Author contributions}

301 The study was conceived and designed by C.A.G. and M.C.T.G. Supporting materials

302 and analysis tools were provided by C.A.G. Experimental data were collected and

303 processed by M.C.T.G. The single-phase $1 \mathrm{fp}$ model was conceived by D.R.V. All the

304 authors contributed writing the paper.

305

\section{Disclosure statement}

307 The authors declare that they have no competing interest.

\section{References}

3091 Burnley. M., Doust, J.H. \& Vanhatalo, A. A 3-min all-out test to determine peak

310 oxygen uptake and the maximal steady state. Med. Sci. Sports Exerc. 38, 1995-2003.

311 https://doi.org/10.1249/01.mss.0000232024.06114.a6 (2006).

3122 Vanhatalo, A., Doust, J.H. \& Burnley, M. Determination of critical power using a 3-

313 min all-out cycling test. Med. Sci. Sports Exerc. 39, 548-555.

314 https://doi.org/10.1249/mss.0b013e31802dd3e6 (2007).

3153 Jones, A.M., Vanhatalo, A., Burnley, M., Morton, R.H. \& Poole, D.C.. Critical power:

316 implications for the determination of VO2 max and exercise tolerance. Med. Sci. Sports

317 Exerc. 42, 1876-1890. https://doi.org/10.1249/MSS.0b013e3181d9cf7f (2010).

3184 Vanhatalo, A. et al. The mechanistic bases of the power-time relationship: muscle

319 metabolic responses and relationships to muscle fibre type. J. Physiol. 594, 4407-4423.

320 https://physoc.onlinelibrary.wiley.com/doi/full/10.1113/JP271879 (2016).

3215 Bertuzzi, R. et al. GEDAE-LaB: A free software to calculate the energy system

322 contributions during exercise. PLOS ONE 11, e0145733.

323 https://doi.org/10.1371/journal.pone.0145733 (2016).

3246 Margaria, R., Edwards, H.T. \& Dill, D.B. The possible mechanisms of contracting 325 and paying the oxygen debt and the role of lactic acid in muscular contraction. Am. J.

326 Physiol. 106, 689-715. https://doi.org/10.1152/ajplegacy.1933.106.3.689 (1933). 
3277 Zagatto, A.M.R. et al. 3-min all-out effort on cycle ergometer is valid to estimate the 328 anaerobic capacity by measurement of blood lactate and excess post-exercise oxygen 329 consumption. Eur. J. Sport Sci. 19, 645-652.

330 https://doi.org/10.1080/17461391.2018.1546338 (2018).

3318 Ozyener, F., Rossiter, H.B., Ward, S.A. \& Whipp, B.J. Influence of exercise intensity 332 on the on- and off-transient kinetics of pulmonary oxygen uptake in humans. J. Physiol. 333 533, 891-902. https://doi.org/10.1111/j.1469-7793.2001.t01-1-00891.x (2001).

3349 Åstrand, P.O., Rodahl, K., Dahl, H.A. \& Strømme, S.B. Textbook of Work

335 Physiology. (Human Kinetics, 2003).

33610 Powers, S.K. \& Howley, E.T. Exercise Physiology. (McGraw-Hill, 1996).

33711 Short, K.R. \& Sedlock, D.A. Excess postexercise oxygen consumption and recovery 338 rate in trained and untrained subjects. J. Appl. Physiol. 83, 153-159.

339 https://doi.org/10.1152/jappl.1997.83.1.153 (1997).

34012 Stupnicki, R., Gabryś, T., Szmatlan-Gabryś, U. \& Tomaszewski, P. Fitting a single341 phase model to the post-exercise changes in heart rate and oxygen uptake. Physiol. Res. 342 59, 357-362. https://www.biomed.cas.cz/physiolres/pdf/59/59_357.pdf (2010.)

34313 Pierpont, G.L., Stolpman, D.R. \& Gornick, C.C. Heart rate recovery post-exercise as 344 an index of parasympathetic activity. J. Autonom. Nerv. Syst. 80, 169-174.

345 https://www.ncbi.nlm.nih.gov/pubmed/10785283 (2000).

34614 Mata, F. Evaluation of horse fitness for exercise: the use of a Logit-Log function to 347 model horse postexercise heart rate recovery. J. Equine Vet. Sci. 34, 1055-1058.

348 Online: http://dx.doi.org/10.1016/j.jevs.2014.06.004 (2014).

34915 Gama, M.C.T., dos Reis, I.G.M., Sousa, F.A.B. \& Gobatto. C.A. The 3-min all-out 350 test is valid for determining critical power but not anaerobic work capacity in tethered 351 running. PLoS ONE 13, e0192552. http://dx.doi.org/10.1371/journal.pone.0192552 352 (2018). 


\section{$354 \quad$ Figure captions}

355 Figure 1. Plots illustrating the effect of varying $k$ (or $f$ ) for a fixed value of the half-

356 recovery time $\tau_{1 / 2}=\ln 2$, according to the $f$-single-phase model. The zoom inserted

357 illustrates the inclination $(\mathrm{d} X / \mathrm{d} t)_{t=0}=-k(1-f)$ at $t=0$ being less abrupt as $f$ increases.

358 The strait dash lines are tangent to the curves at $t=\tau_{1 / 2}$ so their inclinations $(\mathrm{d} X / \mathrm{d} t)_{t=\tau_{1 / 2}}$

$359=-(k / 2)(1-f / 2)$ are more abrupt as $f$ (or equivalently $k$ ) increases.

360

361 Figure 2. Fitting the $f$-single-phase model (full lines) to the experimental data (points).

362 The inserts show residuals of the fitting.

363

364 Figure 3. (a) Illustration of fitting the $f 1 \mathrm{p}$ model and the Stu model to the experimental

365 data of the subject 8 . The inserts show residuals of the fittings. (b) Reduced residual

366 sum of square $\left(\chi^{2}\right)$ and adjusted $R$-square $(R)$ from fitting both models to the set of

367 experimental data. (c) Normal Gaussian distributions of the half-recovery times $\tau_{1 / 2}$ and

$368 T$ projected using the mean values $\left\langle\tau_{1 / 2}\right\rangle$ and $\langle T\rangle$ and the respective standard deviations

$369(\sigma)$.

370

371 Figure 4. (a) Reduced residual sum of square $\left(\chi^{2}\right)$ and adjusted $R$-square $(R)$ from

372 fitting the $f 1 \mathrm{p}$ model and the standing biphasic two-exponential model to the

373 experimental data. (b) Normal Gaussian distributions for the half-recovery times

374 projected using the mean value $\left\langle\tau_{1 / 2}\right\rangle$ (for the $f 1 \mathrm{p}$ model), and the mean values "fast"

$375\left\langle\tau_{1 / 2}{ }^{(1)}\right\rangle$ and "slow" $\left\langle\tau_{1 / 2}{ }^{(2)}\right\rangle$ (for the standard biphasic two-exponential model), with the

376 respective standard deviations $(\sigma)$.

378 Figure 5. Parameters and other quantities evaluated as a function of the parameter $f$ for

379 the $f 1 \mathrm{p}$ model. (a) $\tau_{1 / 2}$ : the linear fitting $\tau_{1 / 2}=0.644-0.0066 \times f$ supporting that $\tau_{1 / 2}$ is

380 practically a constant $\left(\tau_{1 / 2}=\left\langle\tau_{1 / 2}\right\rangle=0.641 \mathrm{~min}\right)$. (b) $k$ : it was well fitted by $k=$

$381\left(1 / \tau_{1 / 2}\right) \ln [(2-f) /(1-f)]$ (from equation (4)) with constant $\tau_{1 / 2}=0.636 \min (\sigma=0.022$

$382 \mathrm{~min})$. (c) $X_{\text {integ: }}$ it was reasonably fitted by equation (7) with constant $\tau_{1 / 2}=0.646$ min $(\sigma$

$383=0.122 \mathrm{~min})$. (d) $\tau_{\mathrm{Q}}$ : it was reasonably fitted by equation (12) with constant $\tau_{1 / 2}=$

$3840.648 \min (\sigma=0.135 \mathrm{~min})$. 
386 Table 1. Fitted parameters for the $f 1 \mathrm{p}$ model, the Stu model, and the standing biphasic

387 two-exponential model. Numbers between the brackets are standard errors estimated by 388 the fitting.

\begin{tabular}{|c|c|c|c|c|c|}
\hline \multirow[b]{2}{*}{ Subject } & \multicolumn{5}{|c|}{$f 1 \mathrm{p}$ model } \\
\hline & $\begin{array}{l}V_{\text {O2peak }} \\
(\mathrm{ml} / \mathrm{min} \cdot \mathrm{kg})\end{array}$ & $\begin{array}{l}V_{\text {O2rest }} \\
(\mathrm{ml} / \mathrm{min} \cdot \mathrm{kg})\end{array}$ & $\begin{array}{l}k \\
\left(\min ^{-1}\right)\end{array}$ & $\begin{array}{l}\tau_{1 / 2} \\
(\min )\end{array}$ & $f$ \\
\hline 1 & $29.9(0.8)$ & $9.09(0.19)$ & $0.99(0.06)$ & $0.700(0.045)$ & 0 \\
\hline 2 & $36.8(0.5)$ & $7.83(0.09)$ & $1.25(0.04)$ & $0.554(0.015)$ & 0 \\
\hline 3 & $27.6(0.8)$ & $7.84(0.14)$ & $1.02(0.16)$ & $0.678(0.057)$ & $0.01(0.22)$ \\
\hline 4 & $40.3(0.9)$ & $8.70(0.16)$ & $1.15(0.12)$ & $0.641(0.039)$ & $0.08(0.19)$ \\
\hline 5 & $41.9(1.2)$ & $11.1(0.2)$ & $1.13(0.11)$ & $0.657(0.052)$ & $0.09(0.20)$ \\
\hline 6 & $45.8(1.2)$ & $9.73(0.19)$ & $2.00(0.22)$ & $0.610(0.037)$ & $0.58(0.10)$ \\
\hline 7 & $37.8(0.7)$ & $9.45(0.12)$ & $2.71(0.23)$ & $0.658(0.024)$ & $0.73(0.06)$ \\
\hline 8 & $35.1(0.9)$ & $9.04(0.15)$ & $2.55(0.26)$ & $0.737(0.037)$ & $0.82(0.04)$ \\
\hline 9 & $28.5(1.0)$ & $9.30(0.17)$ & $2.82(0.40)$ & $0.689(0.057)$ & $0.83(0.03)$ \\
\hline \multirow[t]{3}{*}{10} & $33.0(1.2)$ & $6.97(0.19)$ & $3.58(0.49)$ & $0.574(0.038)$ & $0.85(0.02)$ \\
\hline & \multicolumn{5}{|c|}{ Stu model } \\
\hline & $\begin{array}{l}V_{\text {O2peak }} \\
(\mathrm{ml} / \mathrm{min} \cdot \mathrm{kg})\end{array}$ & $\begin{array}{l}V_{\text {O2rest }} \\
(\mathrm{ml} / \mathrm{min} \cdot \mathrm{kg})\end{array}$ & $\alpha$ & $\begin{array}{l}T \\
(\min )\end{array}$ & \\
\hline 1 & $32.0(1.5)$ & $7.08(0.61)$ & $1.08(0.13)$ & $0.622(0.077)$ & \\
\hline 2 & $36.5(0.6)$ & $6.81(0.16)$ & $1.46(0.06)$ & $0.554(0.022)$ & \\
\hline 3 & $25.9(0.7)$ & $7.50(0.20)$ & $1.84(0.15)$ & $0.775(0.046)$ & \\
\hline 4 & $37.6(0.8)$ & $8.22(0.21)$ & $1.89(0.11)$ & $0.728(0.031)$ & \\
\hline 5 & $39.0(0.9)$ & $10.7(0.3)$ & $1.95(0.15)$ & $0.759(0.040)$ & \\
\hline 6 & $42.9(0.9)$ & $9.25(0.23)$ & $2.19(0.15)$ & $0.665(0.028)$ & \\
\hline 7 & $35.8(0.5)$ & $9.21(0.13)$ & $2.43(0.13)$ & $0.597(0.018)$ & \\
\hline 8 & $33.9(0.7)$ & $8.62(0.18)$ & $2.41(0.17)$ & $0.748(0.030)$ & \\
\hline 9 & $27.8(0.8)$ & $9.00(0.21)$ & $2.39(0.29)$ & $0.696(0.047)$ & \\
\hline \multirow[t]{3}{*}{10} & $31.9(1.0)$ & $6.72(0.20)$ & $2.63(0.25)$ & $0.576(0.030)$ & \\
\hline & \multicolumn{5}{|c|}{ two-exponential model } \\
\hline & $\begin{array}{l}A_{1} \\
(\mathrm{ml} / \mathrm{min} \cdot \mathrm{kg})\end{array}$ & $\begin{array}{l}\tau_{1} \\
(\min )\end{array}$ & $\begin{array}{l}A_{2} \\
(\mathrm{ml} / \mathrm{min} \cdot \mathrm{kg})\end{array}$ & $\begin{array}{l}\tau_{2} \\
(\min )\end{array}$ & \\
\hline 1 & $16(2)$ & $0.47(0.09)$ & $8.3(1.7)$ & $2.9(0.5)$ & \\
\hline 2 & $26(1)$ & $0.57(0.04)$ & $6.0(1.0)$ & $3.3(0.4)$ & \\
\hline 3 & $18(2)$ & $0.85(0.11)$ & $2.2(1.7)$ & $4.4(2.7)$ & \\
\hline 4 & $29(5)$ & $0.79(0.12)$ & $3.7(5.5)$ & $2.4(1.8)$ & \\
\hline 5 & $29(2)$ & $0.77(0.08)$ & $4.6(1.8)$ & $5.1(1.9)$ & \\
\hline 6 & $37(2)$ & $0.69(0.06)$ & $2.8(1.9)$ & $4.4(2.8)$ & \\
\hline 7 & $31(1)$ & $0.65(0.03)$ & $0.8(0.6)$ & $9.9(9.7)$ & \\
\hline 8 & $28(1)$ & $0.76(0.06)$ & $3.1(0.9)$ & $10.1(4.8)$ & \\
\hline 9 & $20(1)$ & $0.74(0.10)$ & $2.3(1.3)$ & $7.1(4.9)$ & \\
\hline 10 & $29(1)$ & $0.59(0.06)$ & $2.6(1.1)$ & $7.2(4.1)$ & \\
\hline
\end{tabular}


Figures

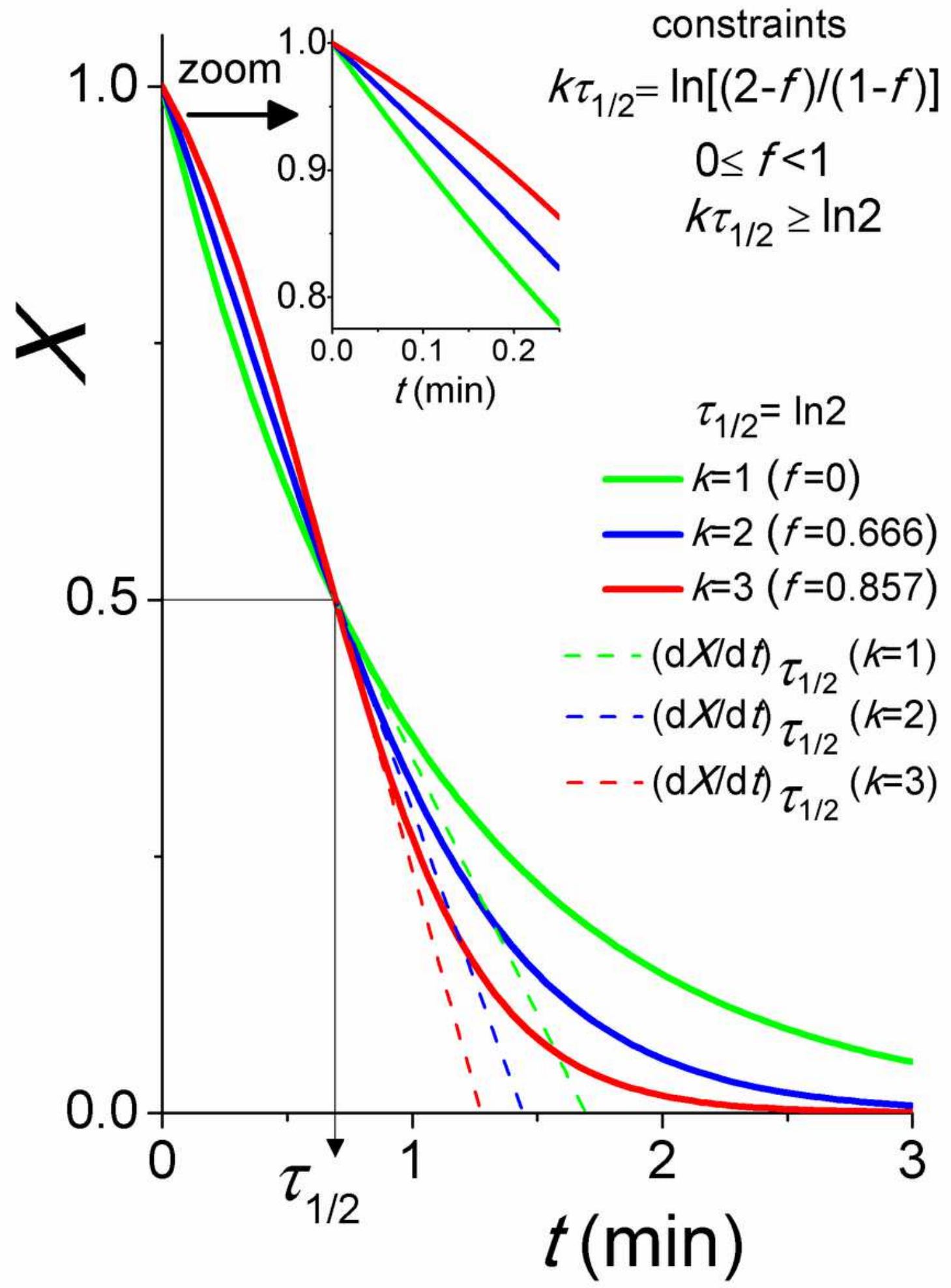

Figure 1

Plots illustrating the effect of varying $k$ (or $f$ ) for a fixed value of the half-recovery time $t 1 / 2=\ln 2$, according to the $f$-single-phase model. The zoom inserted illustrates the inclination $(d X / d t) t=0=-k(1-f)$ at 
$t=0$ being less abrupt as $f$ increases. The strait dash lines are tangent to the curves at $t=t 1 / 2$ so their inclinations $(\mathrm{dX} / \mathrm{dt}) \mathrm{t}=\mathrm{t} 1 / 2=-(\mathrm{k} / 2)(1-\mathrm{f} / 2)$ are more abrupt as $\mathrm{f}$ (or equivalently $\mathrm{k})$ increases.

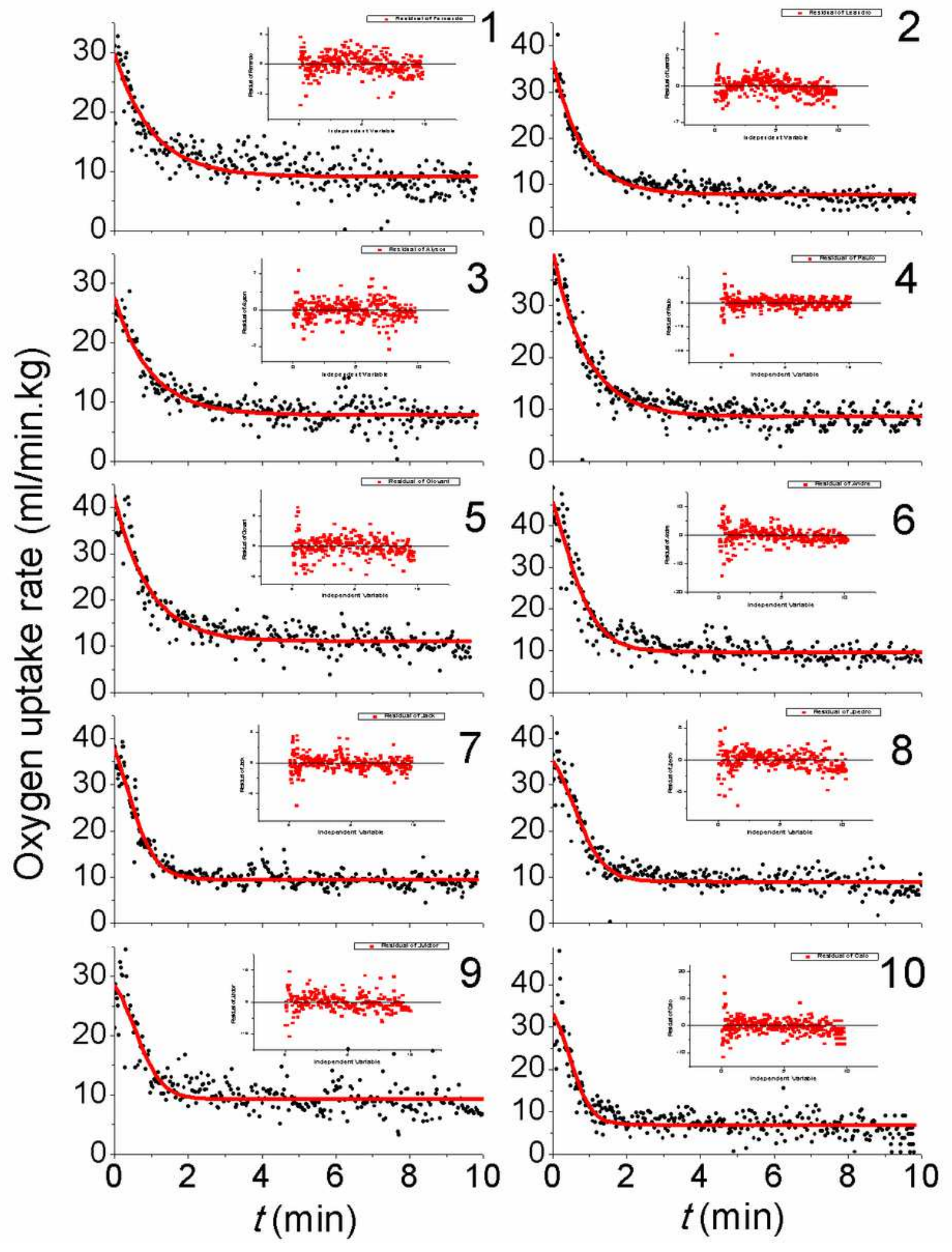

Figure 2

Fitting the f-single-phase model (full lines) to the experimental data (points). The inserts show residuals of the fitting. 

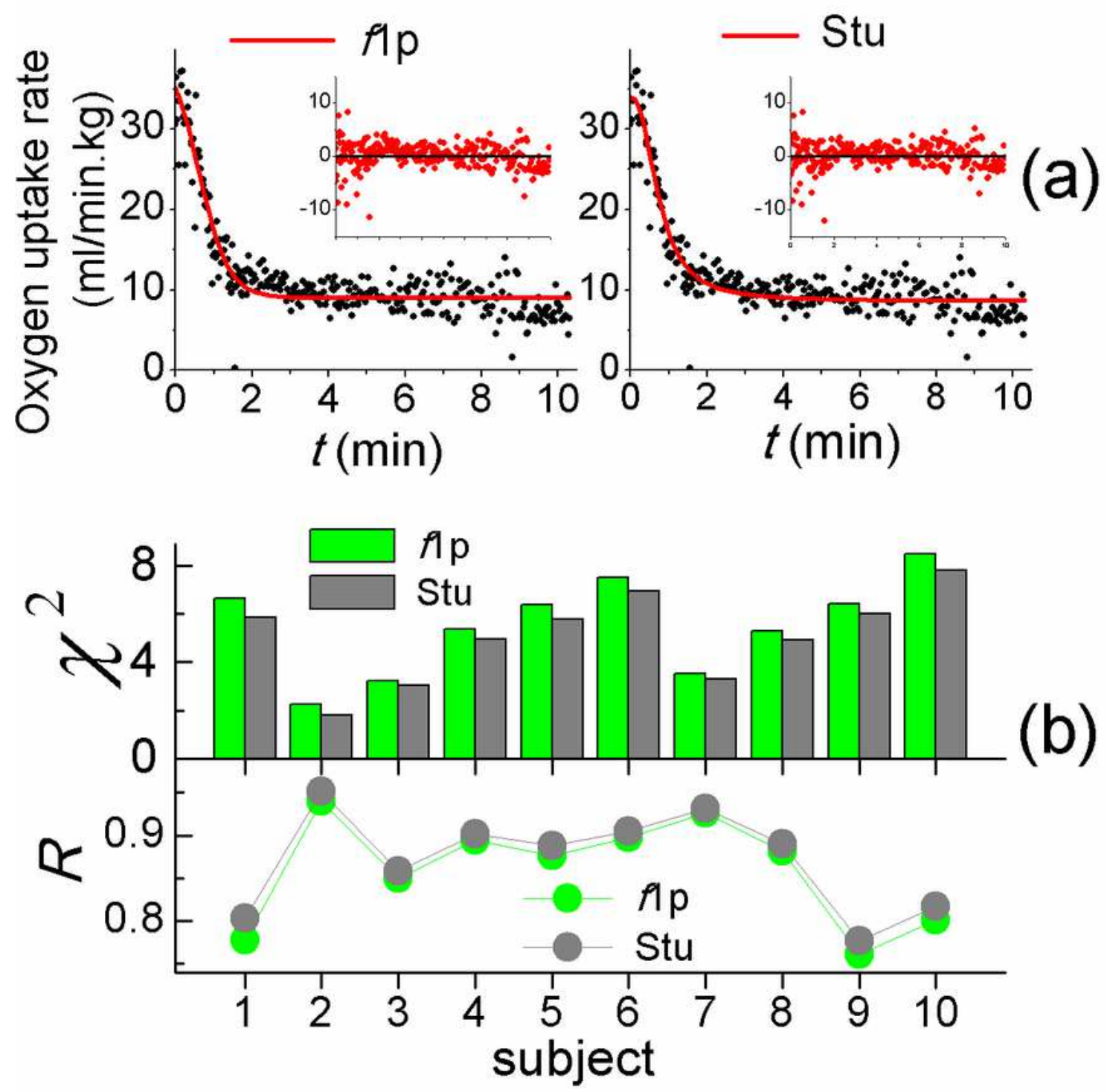

(b)

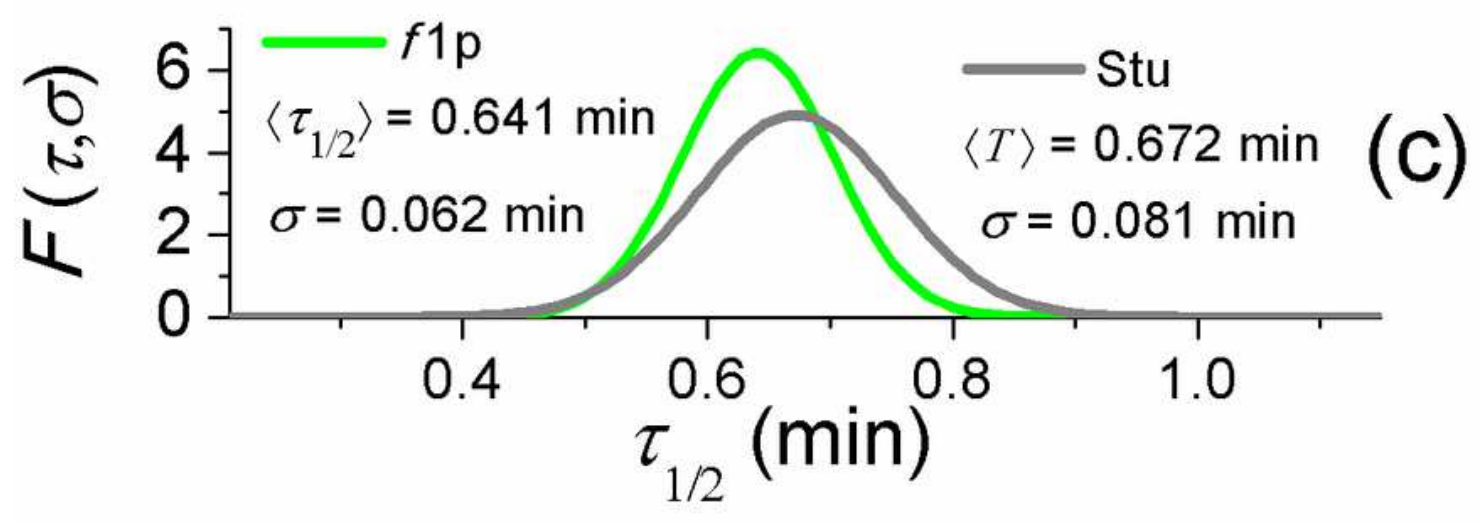

Figure 3

Please see the Manuscript Doc file for the complete figure caption. 

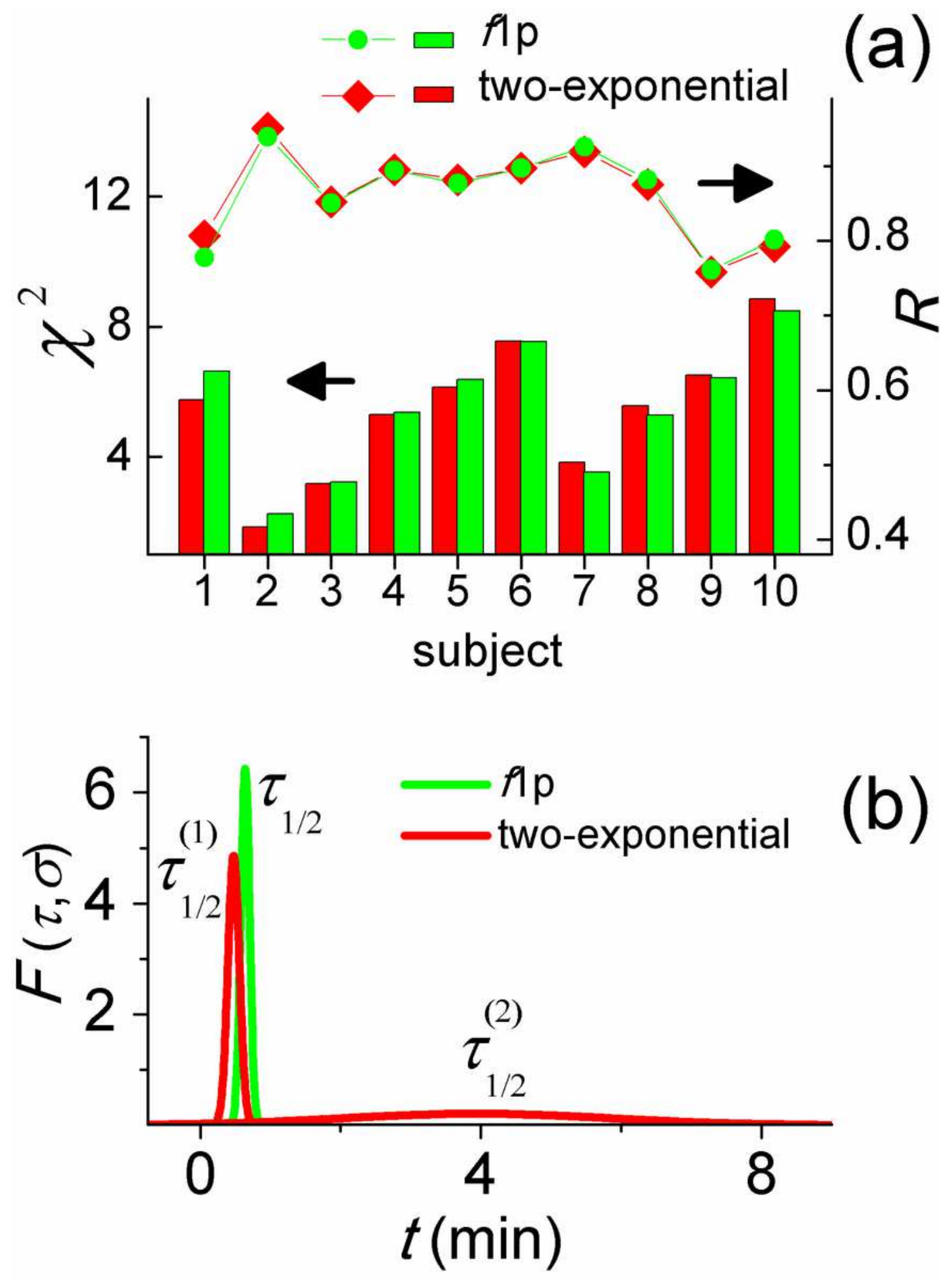

Figure 4

Please see the Manuscript Doc file for the complete figure caption. 


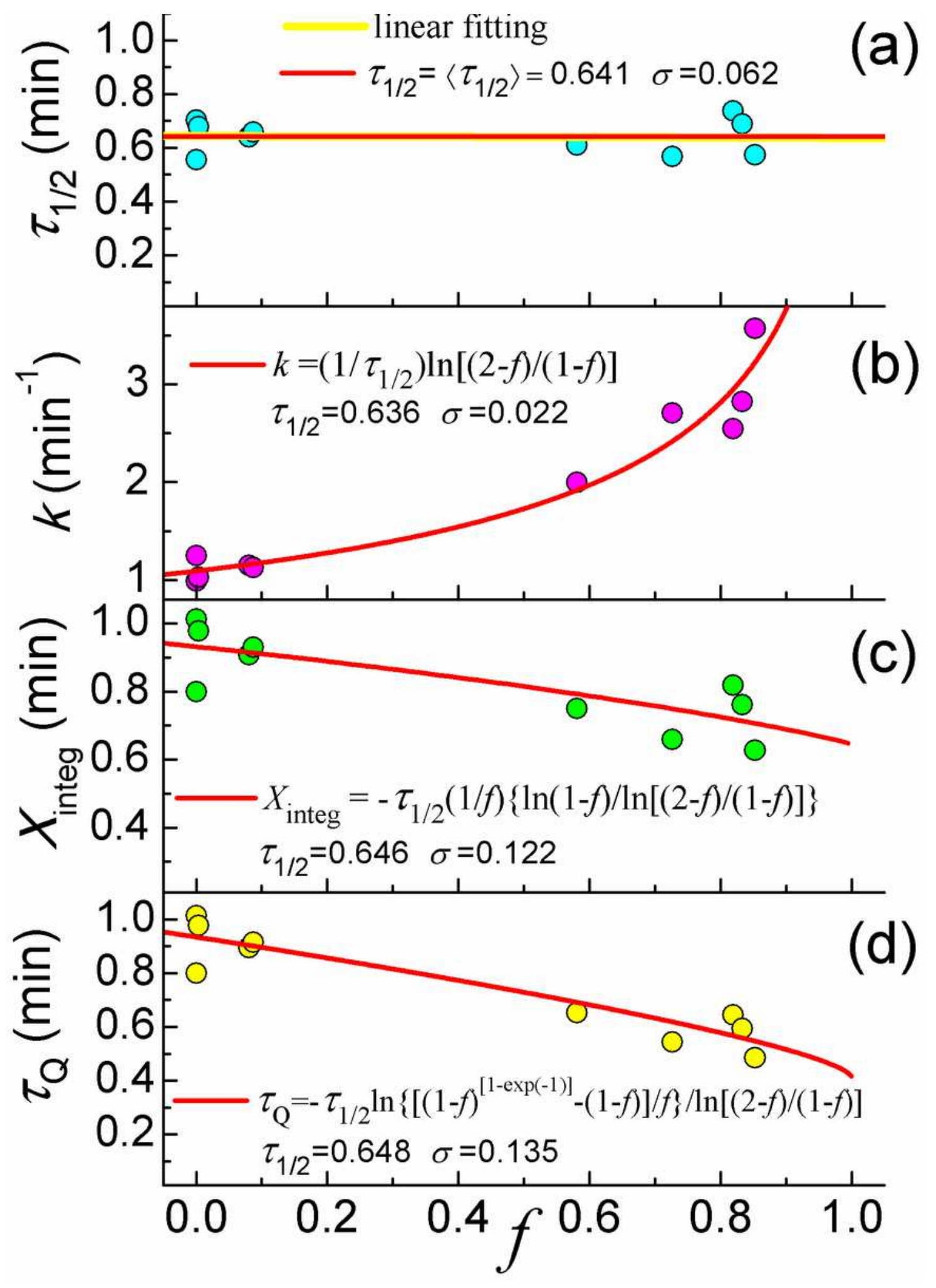

Figure 5

Please see the Manuscript Doc file for the complete figure caption. 